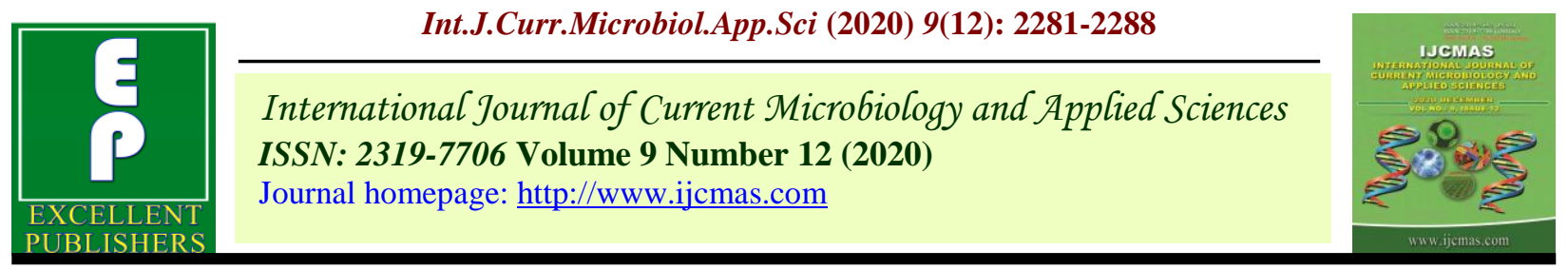

Original Research Article

https://doi.org/10.20546/ijcmas.2020.912.270

\title{
Effect of Shade and Fertigation on Growth and Yield Attributes of Tomato
}

\author{
Kavitha*, M. S. Natarajan and L. Pugalendhi \\ Horticulture, Tamil Nadu Agricultural University, Coimbatore, India \\ *Corresponding author
}

\section{A B S T R A C T}

Keywords

Shade, Fertigation, Water soluble fertilizer, Straight fertilizer, Fruit setting percent, Fruit yield

Article Info

Accepted:

16 November 2020 Available Online:

10 December 2020
Field investigation was conducted at Horticultural College and Research Institute, Tamil Nadu Agricultural University, Coimbatore to elucidate the effects of shade and fertigation on growth, yield and quality of tomato hybrid 'Ruchi'. The experiment was laid out in split plot design with three replications. The main plots were open and shade (35 per cent) and the subplot treatments included three levels (100, 75 and 50 per cent of recommended dose) each of water soluble and straight fertilizers. The yield parameters like number of fruits per plant, fruit weight and polar and equatorial diameters were the highest at 100 per cent WSF under shade. The highest yield per plot (189.6, 208.4 and $203.7 \mathrm{~kg}$ ) and per hectare (99.8, 109.5 and 106.7 tonnes) during seasons I, II and III respectively were observed in the treatment with 100 per cent water soluble fertilizer under shaded condition.

\section{Introduction}

Tomato (Lycopersicon esculentum Mill.) is one of the most popular and versatile vegetable crops grown universally (Cloud, 1974). In India tomato occupies an area of 4.6 lakh hectares with an average productivity of 17.7 tonnes per hectare (Anon., 2002). Globally, about 50 million tonnes of tomatoes are grown annually in the world in over two million hectares of the land but less than 20 per cent of the yield comes from the tropics. In India tomato is predominantly produced in the open field and even sometimes as a rainfed crop. Though productivity of tomato in Indian plains is high, it is reduced largely due to high temperature in summer. Adverse climatic conditions are primarily responsible for the low productivity of tomato (Berry and Uddin, 1988). Incorporating genetic resistance to detrimental effects of high temperature on tomato fruit set has not yielded uniform success and thus cultural methods of overcoming this problem are needed to supply tomato fruit to the existing marketing windows (Russo, 1993). Under these circumstances, use of protected structures would help to increase the productivity and there by production in the region. Practical utility of shade net growing of tomato can be substantiated as it would fetch a premium price in the market where 
there is demand for the fruits during summer is the next best alternative.

Fertigation, a modern agro technique combining water and fertilizer application through irrigation, is one of the most effective and convenient methods of supplying nutrients and water according to the specific requirements of the crop to maintain optimum soil fertility and obtain better quality produce (Shirgure et al., 2000).

\section{Materials and Methods}

The present investigation was carried out at the University Orchard, HC\&RI, Tamil Nadu Agricultural University, Coimbatore. Three field experiments were conducted during 2004-05, two during summer (February- June, 2004 and 2005) and one during kharif (JuneNovember, 2004). The investigation was carried out under controlled environment in shade net house and open condition. The experiments were laid out in split plot design with three replications. Inline drippers were provided in the laterals, with a uniform distance of $60 \mathrm{~cm}$ between the drip emitters.

Treatment details comprises of two main plots (shade $(35 \%)$ and open condition) and with six subplot treatments $(100,75$ and $50 \%$ of recommended dose as water soluble fertilizer and as straight fertilizer). The seedlings were planted in paired row system on both the sides of lateral adopting a spacing of $80 \times 40$ X 60 $\mathrm{cm}$ between pairs, rows and plants, respectively. The recommended dose of $\mathrm{N}$ : P: $\mathrm{K}$ at 250: 250: $250 \mathrm{~kg}$ per hectare was followed in the experiments. Twenty per cent of the recommended $\mathrm{N} \mathrm{P} \mathrm{K}$ was applied as basal through straight fertilizers at the time of planting and the remaining 80 per cent was applied through fertigation. Polyfeed (19:19:19) was used for fertigation with water soluble fertilizer, and urea, superphosphate and muriate of potash were used for fertigation with straight fertilizers. Fertigation was scheduled on alternate days starting from third week after planting as per sub -plot treatments.

Observations on Days to first flowering, Flower clusters per plant, Fruit setting percentage, Polar diameter, Equatorial diameter, Fruits per plant, Fruit weight, Yield per plant and Yield per hectare were recorded.

\section{Results and Discussion}

The influence of shade and fertigation on days taken for first flowering is presented in Table 1. In the interactions, application of 100 per cent water soluble fertilizer under open field condition $\left(\mathrm{M}_{1} \mathrm{~S}_{1}\right)$ registered the lowest number of days for first flowering (27.00, 28.50 and 27.00). The present study revealed that shading prolonged the first flowering compared to open condition. This might be due to increased vegetative growth induced by shade. These results are in accordance with Ramano and Leonardi (1994). Arunkumar (2000) also noticed early flowering in open field than under greenhouse condition. This might be due to prevalence of comparatively higher temperature under open condition. Late flowering under shade may also be due to low solar radiation absorption by plant as interfered by shade cloth.

Application of 100 per cent water soluble fertilizer under shade recorded the highest number of flower clusters per plant with 33.80, 35.60 and 33.96 and the lowest number of flower cluster per plant (17.40, 19.30 and 17.60) was noticed at per cent straight fertilizers under open field condition. Plants under shade showed increased flower clusters per plant. This may be because of the prevalence of favourable conditions required for flowering under shade net. The flower cluster per plant was lower under open condition possibly due to depletion of 
carbohydrate by increased respiration at higher temperature. These findings are in accordance with the results of Suchindra (2002). Generally, flowering was increased with increased levels of fertigation mainly due to early vigour shown by the crop. This could be attributed to the availability of optimum plant nutrients along with sufficient soil moisture for early development of plant parts and root system, which might have enhanced more uptake of nutrients. This is in accordance with Prabhakar et al., (2001) and Meenakshi and Vadivel (2003) (Tables 2-9).

Table.1 Effect of shade and fertigation on days taken for flowering

\begin{tabular}{|c|c|c|c|c|c|c|c|c|c|}
\hline \multirow{2}{*}{ Treatments } & \multicolumn{4}{|c|}{ Season I } & \multicolumn{3}{c|}{ Season II } & \multicolumn{3}{c|}{ Season III } \\
\cline { 2 - 12 } & $\mathbf{M}_{\mathbf{1}}$ & $\mathbf{M}_{\mathbf{2}}$ & $\mathbf{M e a n}$ & $\mathbf{M}_{\mathbf{1}}$ & $\mathbf{M}_{\mathbf{2}}$ & $\mathbf{M e a n}$ & $\mathbf{M}_{\mathbf{1}}$ & $\mathbf{M}_{\mathbf{2}}$ & Mean \\
\hline $\mathrm{S}_{1}$ & 27.00 & 38.00 & 32.50 & 28.50 & 39.50 & 34.00 & 27.00 & 37.50 & 32.25 \\
\hline $\mathrm{S}_{2}$ & 30.50 & 39.00 & 34.75 & 31.00 & 41.00 & 36.00 & 28.50 & 38.00 & 33.25 \\
\hline $\mathrm{S}_{3}$ & 32.00 & 48.00 & 40.00 & 32.50 & 48.50 & 40.50 & 31.50 & 47.50 & 39.50 \\
\hline $\mathrm{S}_{4}$ & 29.00 & 43.00 & 36.00 & 29.50 & 47.00 & 38.25 & 28.50 & 41.50 & 35.00 \\
\hline $\mathrm{S}_{5}$ & 31.50 & 48.50 & 40.00 & 32.00 & 49.50 & 40.75 & 30.50 & 48.00 & 39.25 \\
\hline $\mathrm{S}_{6}$ & 34.50 & 50.50 & 42.50 & 35.00 & 51.00 & 43.00 & 34.00 & 49.00 & 41.50 \\
\hline Mean & 30.75 & 44.50 & 37.63 & 31.42 & 46.09 & 38.75 & 30.00 & 43.58 & 36.79 \\
\hline & $\mathrm{SEd}$ & $\mathrm{CD}(\mathrm{P}=0.05)$ & $\mathrm{SEd}$ & $\mathrm{CD}(\mathrm{P}=0.05)$ & $\mathrm{SEd}$ & $\mathrm{CD}(\mathrm{P}=0.05)$ \\
\hline $\mathrm{S}$ & 0.097 & 0.119 & 0.105 & 0.453 & 0.121 & 0.522 \\
\hline $\mathrm{M}$ & 0.684 & 1.426 & 0.704 & 1.468 & 0.892 & 1.861 \\
\hline $\mathrm{MS}$ & 0.888 & 1.876 & 0.915 & 1.935 & 1.158 & 2.444 \\
\hline $\mathrm{SM}$ & 0.967 & 2.017 & 0.995 & 2.076 & 1.262 & 2.632 \\
\hline
\end{tabular}

Table.2 Effect of shade and fertigation on number of flower cluster per plant

\begin{tabular}{|c|c|c|c|c|c|c|c|c|c|}
\hline \multirow{2}{*}{ Treatments } & \multicolumn{3}{|c|}{ Season I } & \multicolumn{3}{c|}{ Season II } & \multicolumn{3}{c|}{ Season III } \\
\cline { 2 - 12 } & $\mathbf{M}_{\mathbf{1}}$ & $\mathbf{M}_{\mathbf{2}}$ & $\mathbf{M e a n}$ & $\mathbf{M}_{\mathbf{1}}$ & $\mathbf{M}_{\mathbf{2}}$ & $\mathbf{M e a n}$ & $\mathbf{M}_{\mathbf{1}}$ & $\mathbf{M}_{\mathbf{2}}$ & Mean \\
\hline $\mathrm{S}_{1}$ & 30.40 & 33.80 & 32.10 & 33.10 & 35.60 & 34.35 & 31.06 & 33.96 & 32.51 \\
\hline $\mathrm{S}_{2}$ & 27.40 & 27.70 & 27.55 & 27.40 & 29.10 & 28.25 & 26.33 & 27.40 & 26.87 \\
\hline $\mathrm{S}_{3}$ & 19.30 & 19.00 & 19.15 & 20.10 & 21.70 & 20.90 & 19.46 & 19.83 & 19.65 \\
\hline $\mathrm{S}_{4}$ & 26.80 & 31.80 & 29.30 & 29.90 & 34.50 & 32.20 & 27.93 & 32.33 & 30.13 \\
\hline $\mathrm{S}_{5}$ & 23.10 & 26.50 & 24.80 & 25.90 & 28.30 & 27.10 & 26.60 & 26.80 & 26.70 \\
\hline $\mathrm{S}_{6}$ & 17.40 & 18.70 & 18.05 & 19.30 & 20.90 & 20.10 & 17.60 & 19.23 & 18.42 \\
\hline Mean & 24.07 & 26.25 & 25.16 & 25.95 & 28.35 & 27.15 & 24.83 & 26.59 & 25.71 \\
\hline & $\mathrm{SEd}$ & $\mathrm{CD}(\mathrm{P}=0.05)$ & $\mathrm{SEd}$ & $\mathrm{CD}(\mathrm{P}=0.05)$ & $\mathrm{SEd}$ & $\mathrm{CD}(\mathrm{P}=0.05)$ \\
\hline $\mathrm{S}$ & 0.47 & 0.203 & 0.27 & 0.11 & 0.22 & 0.094 \\
\hline $\mathrm{M}$ & 0.76 & 1.59 & 0.65 & 1.36 & 0.46 & 0.97 \\
\hline $\mathrm{MS}$ & 0.98 & 2.06 & 0.84 & 1.76 & 0.60 & 1.25 \\
\hline $\mathrm{SM}$ & 1.07 & 2.24 & 0.92 & 1.92 & 0.66 & 1.37 \\
\hline
\end{tabular}


Table.3 Effect of shade and fertigation on fruit setting percentage in tomato

\begin{tabular}{|c|c|c|c|c|c|c|c|c|c|}
\hline \multirow{2}{*}{ Treatments } & \multicolumn{3}{|c}{ Season I } & \multicolumn{3}{c|}{ Season II } & \multicolumn{3}{c|}{ Season III } \\
\cline { 2 - 11 } & $\mathbf{M}_{\mathbf{1}}$ & $\mathbf{M}_{\mathbf{2}}$ & $\mathbf{M e a n}$ & $\mathbf{M}_{\mathbf{1}}$ & $\mathbf{M}_{\mathbf{2}}$ & Mean & $\mathbf{M}_{\mathbf{1}}$ & $\mathbf{M}_{\mathbf{2}}$ & Mean \\
\hline $\mathrm{S}_{1}$ & 65.13 & 74.84 & 69.99 & 73.82 & 84.93 & 79.38 & 69.92 & 75.93 & 72.92 \\
\hline $\mathrm{S}_{2}$ & 53.68 & 70.31 & 61.99 & 64.24 & 82.31 & 73.28 & 57.56 & 75.23 & 66.39 \\
\hline $\mathrm{S}_{3}$ & 30.82 & 40.46 & 35.64 & 44.60 & 55.78 & 50.19 & 42.54 & 45.46 & 44.00 \\
\hline $\mathrm{S}_{4}$ & 63.60 & 72.53 & 68.07 & 70.16 & 84.69 & 77.43 & 66.67 & 75.46 & 71.18 \\
\hline $\mathrm{S}_{5}$ & 50.64 & 65.22 & 57.93 & 65.52 & 70.15 & 66.34 & 55.25 & 69.05 & 62.15 \\
\hline $\mathrm{S}_{6}$ & 29.61 & 38.92 & 34.27 & 42.77 & 50.63 & 46.70 & 39.77 & 40.63 & 40.20 \\
\hline Mean & 48.91 & 60.38 & 54.65 & 59.69 & 71.42 & 65.55 & 35.29 & 63.66 & 59.47 \\
\hline & $\mathrm{SEd}$ & $\mathrm{CD}(\mathrm{P}=0.5)$ & SEd & $\mathrm{CD}(\mathrm{P}=0.5)$ & $\mathrm{SEd}$ & $\mathrm{CD}(\mathrm{P}=0.5)$ \\
\hline $\mathrm{M}$ & 0.08 & 0.36 & 0.075 & 0.323 & 0.033 & 0.144 \\
\hline $\mathrm{S}$ & 1.67 & 0.49 & 1.592 & 0.321 & 1.086 & 2.267 \\
\hline $\mathrm{MS}$ & 2.16 & 4.52 & 2.056 & 4.296 & 1.404 & 2.929 \\
\hline $\mathrm{SM}$ & 2.37 & 4.95 & 2.252 & 4.697 & 1.537 & 3.207 \\
\hline
\end{tabular}

Table.4 Effect of shade and fertigation on number of fruits per plant

\begin{tabular}{|c|c|c|c|c|c|c|c|c|c|}
\hline \multirow{2}{*}{ Treatments } & \multicolumn{3}{|c|}{ Season I } & \multicolumn{3}{c|}{ Season II } & \multicolumn{3}{c|}{ Season III } \\
\cline { 2 - 11 } & $\mathbf{M}_{\mathbf{1}}$ & $\mathbf{M}_{\mathbf{2}}$ & $\mathbf{M e a n}$ & $\mathbf{M}_{\mathbf{1}}$ & $\mathbf{M}_{\mathbf{2}}$ & $\mathbf{M e a n}$ & $\mathbf{M}_{\mathbf{1}}$ & $\mathbf{M}_{\mathbf{2}}$ & Mean \\
\hline $\mathrm{S}_{1}$ & 40.25 & 55.10 & 47.68 & 57.15 & 60.34 & 58.74 & 49.80 & 60.25 & 55.02 \\
\hline $\mathrm{S}_{2}$ & 36.50 & 35.25 & 35.93 & 45.83 & 52.00 & 48.91 & 35.45 & 46.00 & 40.72 \\
\hline $\mathrm{S}_{3}$ & 20.00 & 22.55 & 21.27 & 39.62 & 41.33 & 40.47 & 27.45 & 37.50 & 32.47 \\
\hline $\mathrm{S}_{4}$ & 39.05 & 53.30 & 46.33 & 55.70 & 59.55 & 57.62 & 48.15 & 55.00 & 51.57 \\
\hline $\mathrm{S}_{5}$ & 36.25 & 34.45 & 35.35 & 48.66 & 52.30 & 58.74 & 38.45 & 45.50 & 41.97 \\
\hline $\mathrm{S}_{6}$ & 19.55 & 20.25 & 19.90 & 35.01 & 40.10 & 37.55 & 34.65 & 35.65 & 35.15 \\
\hline Mean & 31.98 & 36.83 & 34.41 & 46.16 & 50.94 & 48.55 & 38.99 & 46.65 & 42.82 \\
\hline & $\mathrm{SEd}$ & $\mathrm{CD}(\mathrm{P}=0.05)$ & $\mathrm{SEd}$ & $\mathrm{CD}(\mathrm{P}=0.05)$ & $\mathrm{SEd}$ & $\mathrm{CD}(\mathrm{P}=0.05)$ \\
\hline $\mathrm{S}$ & 0.111 & 0.478 & 0.040 & 0.174 & 0.021 & 0.092 \\
\hline $\mathrm{M}$ & 1.061 & 2.213 & 1.163 & 2.427 & 0.767 & 1.599 \\
\hline $\mathrm{MS}$ & 1.374 & 2.887 & 1.503 & 3.137 & 0.990 & 2.067 \\
\hline $\mathrm{SM}$ & 1.501 & 3.131 & 1.645 & 3.432 & 1.085 & 2.263 \\
\hline
\end{tabular}

Table.5 Effect of shade and fertigation on fruit weight (g)

\begin{tabular}{|c|c|c|c|c|c|c|c|c|c|}
\hline \multirow{2}{*}{ Treatments } & \multicolumn{3}{|c|}{ Season I } & \multicolumn{3}{c|}{ Season II } & \multicolumn{3}{c|}{ Season III } \\
\cline { 2 - 11 } & $\mathbf{M}_{\mathbf{1}}$ & $\mathbf{M}_{\mathbf{2}}$ & $\mathbf{M e a n}$ & $\mathbf{M}_{\mathbf{1}}$ & $\mathbf{M}_{\mathbf{2}}$ & $\mathbf{M e a n}$ & $\mathbf{M}_{\mathbf{1}}$ & $\mathbf{M}_{\mathbf{2}}$ & Mean \\
\hline $\mathrm{S}_{1}$ & 60.35 & 65.07 & 62.71 & 50.40 & 62.50 & 56.45 & 58.75 & 62.50 & 60.62 \\
\hline $\mathrm{S}_{2}$ & 47.05 & 55.28 & 51.15 & 49.25 & 46.50 & 47.87 & 47.10 & 54.45 & 50.77 \\
\hline $\mathrm{S}_{3}$ & 40.79 & 42.22 & 41.50 & 27.25 & 36.50 & 31.87 & 21.50 & 42.40 & 31.95 \\
\hline $\mathrm{S}_{4}$ & 59.99 & 62.18 & 61.08 & 49.00 & 54.90 & 51.95 & 56.60 & 60.65 & 58.62 \\
\hline $\mathrm{S}_{5}$ & 40.51 & 49.89 & 45.20 & 36.15 & 45.15 & 40.65 & 40.26 & 53.45 & 46.85 \\
\hline $\mathrm{S}_{6}$ & 36.45 & 40.05 & 38.25 & 32.35 & 33.15 & 32.85 & 33.10 & 41.46 & 37.28 \\
\hline Mean & 47.52 & 52.44 & 49.99 & 40.73 & 46.48 & 43.61 & 42.88 & 52.48 & 47.68 \\
\hline & $\mathrm{SEd}$ & $\mathrm{CD}(\mathrm{P}=0.05)$ & $\mathrm{SEd}$ & $\mathrm{CD}(\mathrm{P}=0.05)$ & $\mathrm{SEd}$ & $\mathrm{CD}(\mathrm{P}=0.05)$ \\
\hline $\mathrm{S}$ & 0.0391 & 0.168 & 0.625 & 0.268 & 0.059 & 0.257 \\
\hline $\mathrm{M}$ & 1.503 & 0.134 & 1.054 & 0.200 & 0.861 & 1.796 \\
\hline $\mathrm{MS}$ & 1.940 & 4.050 & 1.363 & 2.850 & 1.113 & 2.329 \\
\hline $\mathrm{SM}$ & 2.125 & 4.43 & 1.491 & 3.112 & 1.218 & 2.540 \\
\hline
\end{tabular}


Table.6 Effect of shade and fertigation on yield per plant $(\mathrm{kg})$ in tomato

\begin{tabular}{|c|c|c|c|c|c|c|c|c|c|}
\hline \multirow{2}{*}{ Treatments } & \multicolumn{3}{|c|}{ Season I } & \multicolumn{3}{c|}{ Season II } & \multicolumn{3}{c|}{ Season III } \\
\cline { 2 - 11 } & $\mathbf{M}_{\mathbf{1}}$ & $\mathbf{M}_{\mathbf{2}}$ & Mean & $\mathbf{M}_{\mathbf{1}}$ & $\mathbf{M}_{\mathbf{2}}$ & $\mathbf{M e a n}$ & $\mathbf{M}_{\mathbf{1}}$ & $\mathbf{M}_{\mathbf{2}}$ & Mean \\
\hline $\mathrm{S}_{1}$ & 2.925 & 3.328 & 3.127 & 2.880 & 3.65 & 3.265 & 2.839 & 3.585 & 3.212 \\
\hline $\mathrm{S}_{2}$ & 1.669 & 1.960 & 1.815 & 1.798 & 1.953 & 1.876 & 1.717 & 1.954 & 1.835 \\
\hline $\mathrm{S}_{3}$ & 0.754 & 0.988 & 0.871 & 0.721 & 0.944 & 0.833 & 0.815 & 0.952 & 0.883 \\
\hline $\mathrm{S}_{4}$ & 2.725 & 3.032 & 2.879 & 2.743 & 3.269 & 3.006 & 2.174 & 3.314 & 2.744 \\
\hline $\mathrm{S}_{5}$ & 1.547 & 1.950 & 1.749 & 1.578 & 1.909 & 1.744 & 1.188 & 1.718 & 1.453 \\
\hline $\mathrm{S}_{6}$ & 0.643 & 0.932 & 0.788 & 0.677 & 0.913 & 0.795 & 1.120 & 0.965 & 1.042 \\
\hline Mean & 1.603 & 2.032 & 1.872 & 1.733 & 2.106 & 1.919 & 1.642 & 2.081 & 1.862 \\
\hline & $\mathrm{SEd}$ & $\mathrm{CD}(\mathrm{P}=0.05)$ & $\mathrm{SEd}$ & $\mathrm{CD}(\mathrm{P}=0.05)$ & $\mathrm{SEd}$ & $\mathrm{CD}(\mathrm{P}=0.05)$ \\
\hline $\mathrm{S}$ & 0.004 & 0.016 & 0.005 & 0.019 & 0.014 & 0.136 \\
\hline $\mathrm{M}$ & 0.050 & 0.125 & 0.049 & 0.103 & 0.048 & 0.137 \\
\hline $\mathrm{MS}$ & 0.077 & 0.161 & 0.064 & 0.134 & 0.064 & 0.202 \\
\hline $\mathrm{SM}$ & 0.084 & 0.176 & 0.069 & 0.146 & 0.068 & 0.194 \\
\hline
\end{tabular}

Table.7 Effect of shade and fertigation on estimated yield in Tomato $\left(t \mathrm{tha}^{-1}\right)$

\begin{tabular}{|l|c|c|c|}
\hline \multicolumn{1}{|c|}{ Treatments } & Season I & Season II & Season III \\
\hline Open & & & \\
\hline 100 per cent WSF & 89.8 & 99 & 97.1 \\
\hline 75 per cent WSF & 53.3 & 53.1 & 53.1 \\
\hline 50 per cent WSF & 26.6 & 25.5 & 89.7 \\
\hline 100 per cent SF & 82.0 & 88.6 & 46.3 \\
\hline 75 per cent SF & 53.0 & 51.8 & 25.2 \\
\hline 50 per cent SF & 25.2 & 24.7 & 106.7 \\
\hline Shade & & & 58.5 \\
\hline 100 per cent WSF & 99.8 & 109.5 & 28.6 \\
\hline 75 per cent WSF & 58.7 & 58.5 & 99.4 \\
\hline 50 per cent WSF & 29.6 & 28.3 & 51.6 \\
\hline 100 per cent SF & 90.8 & 97.9 & 28.9 \\
\hline 75 per cent SF & 58.4 & 57.1 & \\
\hline 50 per cent SF & 28.1 & 27.5 & \\
\hline
\end{tabular}

Table.8 Effect of shade and fertigation on polar diameter $(\mathrm{cm})$ in tomato

\begin{tabular}{|c|c|c|c|c|c|c|c|c|c|}
\hline \multirow{2}{*}{ Treatments } & \multicolumn{3}{|c}{ Season I } & \multicolumn{3}{c|}{ Season II } & \multicolumn{3}{c|}{ Season III } \\
\cline { 2 - 12 } & $\mathbf{M}_{\mathbf{1}}$ & $\mathbf{M}_{\mathbf{2}}$ & $\mathbf{M e a n}$ & $\mathbf{M}_{\mathbf{1}}$ & $\mathbf{M}_{\mathbf{2}}$ & $\mathbf{M e a n}$ & $\mathbf{M}_{\mathbf{1}}$ & $\mathbf{M}_{\mathbf{2}}$ & Mean \\
\hline $\mathrm{S}_{1}$ & 3.03 & 3.24 & 3.13 & 4.29 & 4.35 & 4.32 & 3.12 & 4.28 & 3.70 \\
\hline $\mathrm{S}_{2}$ & 2.93 & 3.15 & 3.04 & 3.60 & 4.26 & 3.93 & 3.47 & 3.37 & 3.42 \\
\hline $\mathrm{sS}_{3}$ & 2.90 & 3.00 & 2.95 & 3.15 & 3.45 & 3.30 & 3.05 & 3.28 & 3.16 \\
\hline $\mathrm{S}_{4}$ & 2.85 & 3.17 & 3.01 & 3.75 & 3.83 & 3.79 & 3.63 & 3.78 & 3.70 \\
\hline $\mathrm{S}_{5}$ & 2.74 & 3.06 & 2.90 & 3.25 & 3.70 & 3.47 & 2.80 & 3.13 & 2.96 \\
\hline $\mathrm{S}_{6}$ & 2.59 & 2.86 & 2.72 & 3.19 & 3.52 & 3.35 & 2.92 & 3.10 & 3.01 \\
\hline Mean & 2.84 & 3.08 & 2.96 & 3.54 & 3.85 & 3.69 & 3.16 & 3.49 & 3.33 \\
\hline & $\mathrm{SEd}$ & $\mathrm{CD}(\mathrm{P}=0.05)$ & $\mathrm{SEd}$ & $\mathrm{CD}(\mathrm{P}=0.05)$ & $\mathrm{SEd}$ & $\mathrm{CD}(\mathrm{P}=0.05)$ \\
\hline $\mathrm{S}$ & 0.043 & 0.184 & 0.042 & 0.181 & 0.028 & 0.123 \\
\hline $\mathrm{M}$ & 0.077 & 0.159 & 0.077 & 0.161 & 0.051 & 0.108 \\
\hline $\mathrm{MS}$ & 0.108 & 0.263 & 0.108 & 0.262 & 0.072 & 0.176 \\
\hline $\mathrm{SM}$ & 0.108 & 0.226 & 0.109 & 0.228 & 0.073 & 0.152 \\
\hline
\end{tabular}


Int.J.Curr.Microbiol.App.Sci (2020) 9(12): 2281-2288

Table.9 Effect of shade and fertigation on equatorial diameter $(\mathrm{cm})$ in tomato

\begin{tabular}{|c|c|c|c|c|c|c|c|c|c|}
\hline \multirow{2}{*}{ Treatments } & \multicolumn{3}{|c}{ Season I } & \multicolumn{3}{c|}{ Season II } & \multicolumn{3}{c|}{ Season III } \\
\cline { 2 - 12 } & $\mathrm{M}_{1}$ & $\mathrm{M}_{2}$ & Mean & $\mathrm{M}_{1}$ & $\mathrm{M}_{2}$ & Mean & $\mathrm{M}_{1}$ & $\mathrm{M}_{2}$ & Mean \\
\hline $\mathrm{S}_{1}$ & 5.45 & 6.72 & 6.09 & 7.23 & 7.43 & 7.33 & 5.66 & 5.96 & 5.18 \\
\hline $\mathrm{S}_{2}$ & 5.15 & 4.11 & 4.63 & 6.80 & 7.1 & 6.95 & 5.05 & 5.18 & 5.12 \\
\hline $\mathrm{S}_{3}$ & 4.02 & 3.82 & 3.92 & 6.30 & 0.83 & 6.56 & 4.54 & 4.78 & 4.66 \\
\hline $\mathrm{S}_{4}$ & 4.26 & 5.98 & 5.12 & 6.87 & 6.95 & 6.91 & 5.24 & 5.73 & 5.49 \\
\hline $\mathrm{S}_{5}$ & 3.78 & 3.75 & 3.77 & 6.63 & 6.25 & 6.44 & 3.86 & 4.54 & 4.20 \\
\hline $\mathrm{S}_{6}$ & 3.22 & 3.55 & 3.39 & 5.80 & 5.10 & 5.45 & 3.73 & 3.66 & 3.69 \\
\hline Mean & 4.31 & 4.66 & 4.49 & 6.60 & 6.61 & 6.61 & 4.68 & 4.68 & 4.83 \\
\hline & $\mathrm{SEd}$ & $\mathrm{CD}(\mathrm{P}=0.05)$ & $\mathrm{SEd}$ & $\mathrm{CD}(\mathrm{P}=0.05)$ & $\mathrm{SEd}$ & $\mathrm{CD}(\mathrm{P}=0.05)$ \\
\hline $\mathrm{S}$ & 0.055 & 0.238 & 0.069 & $\mathrm{NS}$ & 0.035 & 0.152 \\
\hline $\mathrm{M}$ & 0.123 & 0.257 & 0.139 & 0.291 & 0.077 & 0.162 \\
\hline $\mathrm{MS}$ & 0.169 & 0.392 & 0.193 & 0.459 & 0.106 & 0.247 \\
\hline $\mathrm{SM}$ & 0.174 & 0.364 & 0.197 & 0.411 & 0.109 & 0.228 \\
\hline
\end{tabular}

Among the interactions, 100 per cent water soluble fertilizer under shade $\left(\mathrm{M}_{2} \mathrm{~S}_{1}\right)$ registered the highest fruit setting percentage of $74.84,84.93$ and 75.93 per cent. The treatments differed significantly among themselves. The fruit set was higher under shade than open condition, possibly be due to excessive style elongation and exertion of stigma, which is a common feature in tomato grown at high temperature. This is in conformity with the findings of Rick and Demprey (1969), Anbu (1978) and Kalloo (1989). High temperature induces the flower abscission and reduces the fruit set thereby reducing the yield. Under open condition, the number of fruits per plant was decreased due to high temperature, which would have increased the rate of abortion (Charles and Harris, 1972).

The interaction $\mathrm{M}_{2} \mathrm{~S}_{1}$ recorded the highest number of fruits per plant per $(55.10,60.34$ and 60.25), which was closely followed by $\mathrm{M}_{2} \mathrm{~S}_{4}$ with 53.30, 59.55 and 55.00 respectively. According to Marschner (1983), a balanced supply of nitrogen promotes the translocation of phytohormones to the shoot that probably induces the flower and fruit initiation. The results showed that application of 100 per cent water soluble fertilizer recorded the highest number of fruits. This might be due to higher production of photosynthates resulting in increased production of fruits. These results are in accordance with the findings of Yadav and Bhupender Singh (1991), Locascio and Smajstrla (1995) and Salvadore et al., (1997).

The interaction $\mathrm{M}_{2} \mathrm{~S}_{1}$ (100 per cent water soluble fertilizer under shade) registered the highest fruit weight of 65.07, 62.50 and 62.50 $\mathrm{g}$ during seasons I, II and III respectively. The fruits harvested under shade had more weight than under open condition. The fruit weight is highly influenced by growing conditions and reduction in fruit size under open might be due to the reduction in the supply of assimilates to developing sink caused by exhaustion through excess respiration (Cockshell et al., 1992).

The highest fertigation level resulted in the highest average single fruit weight, which could be attributed to the nature of interaction of physiological and growth parameters by way of increased dry matter production. These results are in conformity with that of Felipe and Casanova (2000) in tomato.

Combination of 100 per cent water soluble fertilizer and shade $\left(\mathrm{M}_{2} \mathrm{~S}_{1}\right)$ recorded the highest yield of 3.328, 3.650 and $3.585 \mathrm{~kg}$ per 
plant. The shade and fertigation levels singly and in combination significantly influenced the yield. The highest yield of 99.8, 109.5 and $106.7 \mathrm{t} \mathrm{ha}^{-1}$ was obtained at 100 per cent water soluble fertilizer under shade, The favourable microclimate coupled with 100 per cent nutrients supplied through fertigation could be attributed to the best performance of the treatments. The yield is a complex trait, which is dependent on many contributing traits. The best results obtained in respect of the growth parameters like plant height, number of laterals per plant and dry matter production could be attributed to the highest yield. The fruit characters like number of fruits, fruit weight and polar and equatorial diameters, which registered the highest values, would have directly influenced the yield. The yield under open condition was less because of the fact that mobilization and translocation of nutrients and photosynthates to the developing parts was much more reduced at high temperature experienced in open condition than under shade. These results are in accordance with that of Arunkumar (2000) and Suchindra (2002).

During all the three seasons, application of 100 per cent water soluble fertilizer under shade condition $\left(\mathrm{M}_{2} \mathrm{~S}_{1}\right)$ obtained the highest polar diameter $3.24,4.35$ and $4.28 \mathrm{~cm}$ and equatorial diameter of $6.72,7.43$ and 5.96 during seasons I, II and III respectively. These traits registered the highest values at the highest fertigation level with water soluble fertilizers. These results are in agreement with that of Lata and Singh (1993) in chilli.

\section{References}

Anbu, S. 1978. Studies on heterosis in tomato (Lycopersicon esculentum Mill.) M.Sc. (Ag.) Thesis, Tamil Nadu Agricultural University, Coimbatore.

Arunkumar, R.2000. Evaluation of certain tomato (Lycopersicon esculentum Mill.) genotypes under greenhouse and open conditioning. M.Sc. thesis, Tamil Nadu Agricultural University, Coimbatore.

Charles, W.B. and R.E. Harris. 1972. Tomato fruit set at high and low temperatures. Can. J. Plant. Sci., 52: 497-506.

Cockshell, K.E., C.J. Graves and C.R.J. Cave. 1992. The influence of shading on yield of glasshouse tomatoes. J. Hort. Sci., 67: 11-24.

Felipe, E.F. and O.E. Casanova. 2000. Nitrogen, phosphorus and potassium fertilization in tomato (Lycopersicon esculentum Mill.) in the alluvial bank soils of the Guarico river. Revista Unillez de Ciencia y Technologia, 17: 21-44.

Kalloo, G. 1989. Tomato (Lycopersicon esculentum Mill.) Ind. Hort., 33 \& 34: 12-15.

Lata, S. and R.P. Singh. 1993. Effect of nitrogen level and growth regulators on growth, yield and quality of chilli (Capsicum annuum L.) variety Pant C1. Veg. Sci., 20: 40-43.

Locascio, S.J. and A.G. Smajstrla. 1992. Water and N-K - application timing for drip irrigated tomato. Proc. of the Int. Amer. Soc. For Trop. Hort., 36: 104111.

Marschner, H. 1983. Introduction to the mineral nutrition of plants. In : Handb. Pflphysiol., 154: 31-38.

Meenakshi, N. and E. Vadivel. 2002. Growth and productivity of hybrid bitter gourd (Nomoridica charantia L.) $\mathrm{COB} \mathrm{GOH} \mathrm{-}$ I under different macro and micro nutrient fertigation levels. Ph.D. (Hort.) thesis submitted to Tamil Nadu Agricultural University, Coimbatore.

Prabhakar, M. Vijaya - Savanur, Naik C.L., Savanur, V. 2001. Fertigation studies in hybrid tomato. South Indian Horticulture, 49: 98-100.

Rick, C.M. and W.H. Demprey. 1969. Position of the stigma in relation to fruit 
setting I tomato. Bot. Coz., 130: 180186.

Ramano, D. and C. Leonardi. 1994. The response of tomato and egg plant to different minimum air temperatures. Acta Hort., 366: 57-63.

Salvadore, J.L., G.J. Hochmuth, M.R. Fred, S.M. Olson, A.G. Smajstrala, Ed. A. Honlon. 1997. Nitrogen and potassium application scheduling effects on drip irrigated tomato yield and leaf tissue analysis. Hort. Sci., 3: 230-235.
Suchindra, R. 2002. Studies on evaluation of tomato (Lycopersicon esculentum Mill.) hybrids under four different growing environments for yield and quality characters. M.Sc. thesis, Horticultural College and Research Institute, Periyakulam.

Yadav, A.C. and Bhupender Singh. 1991. Water stress in vegetable production - a review. Agric. Rev., 12:177-184.

\section{How to cite this article:}

Kavitha, M. S. Natarajan and Pugalendhi, L. 2020. Effect of Shade and Fertigation on Growth and Yield Attributes of Tomato. Int.J.Curr.Microbiol.App.Sci. 9(12): 2281-2288.

doi: https://doi.org/10.20546/ijcmas.2020.912.270 